\title{
A search for Gram-negative enteric micro-organisms in acute anterior uveitis: association of klebsiella with recent onset of disease, HLA-B27, and B7 CREG
}

\author{
LAURINE WHITE, RICHARD MCCOY, BRIAN TAIT, * AND ROLAND EBRINGER \\ From the Department of Medicine, University of Melbourne, Austin Hospital; the Uveitis \\ Research Clinic, Royal Victorian Eye and Ear Hospital; and the *Tissue Typing Laboratory, \\ Royal Melbourne Hospital, Australia
}

SUMMARY Faecal samples obtained from patients with acute anterior uveitis (AAU) and healthy controls unconnected with hospitals were examined for the presence of Gram-negative microorganisms reported to be associated with AAU. There was an increased recovery of Klebsiella spp. from AAU patients seen in the first and in the second week after onset of symptoms when compared with patients presenting three to four weeks after onset of symptoms $(\mathrm{p}<0.025$ and $p<0.05$ respectively) or with healthy control subjects $(\mathrm{p}<0.005$ and $\mathrm{p}<0.05$ respectively). By the third to fourth week there was no difference in klebsiella recovery between AAU patients and controls. The increased faecal recovery of klebsiella in the first two weeks was predominantly in HLA-B27 positive patients or patients possessing antigens which cross-react with B27, namely, HLA-B7 CREG. The patients with faecal cultures positive for klebsiella had a higher mean ESR than patients with negative faecal cultures $(p<0.05)$. Although there was an increase in recovery of faecal klebsiella from AAU patients with spondylarthritis when compared with AAU patients without arthritis, the difference was not statistically significant. These results suggest that some klebsiella micro-organisms may play a role in the aetiopathogenesis of AAU associated with HLA-B27 or HLA-B7 CREG.

Acute anterior uveitis (AAU) is most probably a heterogeneous group of disorders, some of which may be produced as a consequence of infection.' AAU following enteric infection with shigella, ${ }^{2}$, salmonella, ${ }^{3}$ and Yersinia enterocolitica ${ }^{4}$ has been described. There are indications that immune mechanisms may play a critical role in the production of AAU, but in the majority of cases the aetiology is unknown. About half of the patients with AAU are found to carry the tissue antigen HLA-B27, and a high proportion of these patients have associated spondylarthritic diseases such as ankylosing spondylitis (AS) or sacroiliitis. ${ }^{67}$ Several studies have reported an increase of HLA-B7

Correspondence to Dr R. Ebringer, Department of Medicine, University of Mclbourne, Austin Hospital, Heidelberg, Victoria 3084, Australia.
CREG antigens in B27 negative AS. (The B7 CREG antigens cross-react serologically with B27 and include B7, Bw22, Bw40, and Bw42.) Two recent studies have also noted an increase of $B 7$ CREG in AAU. ${ }^{x 9}$ Cross-reactivity between some strains of Klebsiella spp. and HLA-B27 positive AS lymphocytes has been reported. ${ }^{1012}$ Increased faecal recovery of Klebsiella pneumoniae during exacerbations of active inflammation of spinal and peripheral joint and eye disease in AS has been observed, ${ }^{1 ?-15}$ though some investigators were unable to confirm this. ${ }^{\text {It }}$ An increased faecal isolation of Klebsiella spp. in AAU associated with AS has also been reported, ${ }^{17}{ }^{18}$ though again not confirmed by others. ${ }^{10}$ The evidence suggests that infection by some strains of Gram-negative micro-organisms, including some Klebsiella spp, may be an aetiological factor in the production of acute inflammation in AS. 
The aim of these investigations was to search for evidence of recent enteric carriage by AAU patients of Gram-negative micro-organisms implicated in the aetiopathogenesis of the HLA-B27 related disorders. The bacteria sought included shigella, salmonella, Yersinia enterocolitica, Campylobacter fetus subsp. jejuni, and Klebsiella spp.

\section{Patients and methods}

Patient selection. Patients presenting with a first attack or a recurrence of AAU to the Royal Victorian Eye and Ear Hospital emergency department were referred to the weekly uveitis research clinic. Patients were seen within the first week of presenting to the eye casualty clinic. Occasionally patients were seen within two weeks. This hospital is the major referral centre in the region for acute ocular emergencies and serves a population of over two million. Because of delay between onset of symptoms and first presentation to the eye emergency department, many patients were seen for the first time in our research clinic three to four weeks after the onset of ocular symptoms. At the initial presentation to the eye casualty department patients received sterile faecal specimen containers with instructions to provide a fresh faecal sample on the day of attendance at the clinic. Apart from this initial presentation, patients did not have any significant hospital exposure prior to our clinical assessment. All patients with AAU who provided faecal samples within the four weeks from onset of symptoms were included in the study.

The patients were divided into three groups: those providing faecal samples within one week of onset of symptoms, group A; those providing faecal samples in the second week after onset of symptoms, group $\mathrm{B}$; and those providing samples in the third and fourth week after onset of symptoms, group C. The patients were examined, and blood was drawn for HLA-A and B tissue typing and erythrocyte sedimentation rate (ESR). Radiographs of the pelvis and spine were performed where indicated by the history or examination. Patients with chronic uveitis (that is, inflammation for more than three months) or patients with posterior uveitis were excluded from the study.

Control subjects. Healthy control subjects were recruited from general practitioner's lists in the Melbourne area. Subjects were examined and single faecal samples collected at the local practitioner clinic. Persons with a history of ocular inflammation, peripheral or spinal arthritis, psoriasis, inflammatory bowel disease, a recent history of significant hospital contact, or antibiotic use in the previous two months were excluded from the control group.
Hospital staff. Thirty-one healthy hospital staff provided single faecal specimens.

Faecal cultures. Faecal specimens were examined for Gram-negative enteric organisms, including known pathogens, by standard methods. Briefly, specimens were cultured on to MacConkey, xylose lysine dehydrogenase (XLD), and inositol-supplemented agar. The latter was included to differentiate Klebsiella spp. from other lactose-fermenters. Faecal specimens were incubated at $37^{\circ} \mathrm{C}$ for 18 hours before examination for inositol fermenting colonies. Salmonellae were sought on bismuth sulphite agar following selenite enrichment.

A small amount of faeces was emulsified in phosphate-buffered saline (PBS) containing 1\% mannitol, stored at $4^{\circ} \mathrm{C}$ for six weeks to enrich for Yersinia enterocolitica, and subcultured on to Hektoen Enteric agar at $25^{\circ} \mathrm{C}$ for 48 hours. Presumptive Klebsiella/Yersinia spp. were identified by the API 20E system.

Statistical analysis was by $2 \times 2 \chi^{2}$ tables, with Yates's correction for small numbers, or Student's $t$ test.

\section{Results}

There were 75 patients and 76 episodes of AAU available for analysis, one patient having two separate attacks. The mean age of the patients was 39.7 years. Thirty-one were female. Fifty-four control subjects were obtained. Their mean age was 40.5 years and 29 were female.

No salmonella, shigella, or Campylobacter fetus spp. jejuni micro-organisms were isolated from either patient or control faecal cultures. Yersinia enterocolitica (serotype not known) was isolated from one patient who had no enteric symptoms.

\section{Klebsiella in $A A U$}

The isolation rate of klebsiella in AAU patients and controls is shown in Fig. 1. There was a significant increase in faecal klebsiella recovery in group A AAU patients during the first week $10 / 25(40 \%)$ when compared with group $C$ patients $1 / 22(5 \%)$ $\left(\chi^{2}=6.35, p<0.025\right)$ or with control subjects $5 / 54$ $(9 \%) \quad\left(\chi^{2}=8.59, p<0.005\right)$. There was a lower, though still significant, increase in recovery of klebsiella in group B AAU patients during the second week $9 / 29$ (31\%) when compared with group C patients $\left(\chi^{2}=4.01, p<0.05\right)$ or with control subjects $\left(\chi^{2}=4.92, p<0.05\right)$. By the third to fourth week there was no difference in the klebsiella recovery rate between AAU patients and controls.

Twelve of the 31 faecal specimens provided by hospital staff grew klebsiella $(39 \%)$. This was a significantly higher isolation rate than the non- 


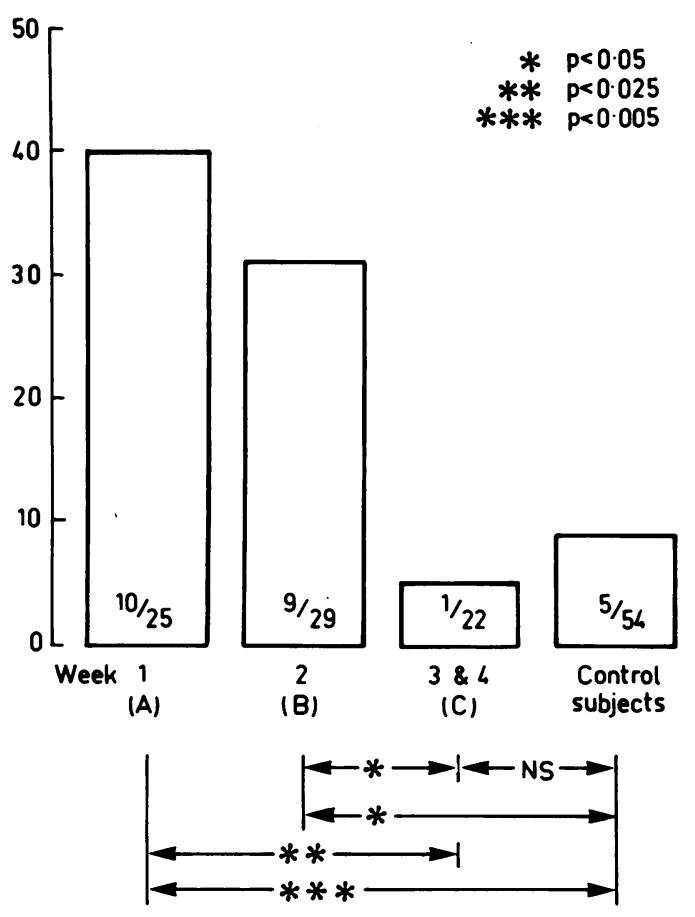

Fig. 1 Percentage faecal isolation rate of Klebsiella spp. in $A A U$ patients one week after onset of symptoms (group $A$ ), two weeks after onset (group B), three to four weeks after onset (group $C$ ), and in healthy non-hospital controls.

hospital control subjects $\left(\chi^{2}=8.91 \mathrm{p}<0.005\right)$ or the group C AAU patients $\left(\chi^{2}=6.37 \mathrm{p}<0.025\right)$.

Klebsiella in HLA-B27, B7 CREG, and non-B7 CREG patients

The results of comparing the faecal isolations from HLA-B7 CREG with non-B7 CREG AAU patients and controls are shown in Fig. 2. Ther was an increased isolation of Klebsiella spp. from HLA-B27 $(50 \%)$ and particularly HLA-B7 CREG (53\%) patients in the first week when compared with HLAB7 CREG negative patients $(22 \%)$, but possibly owing to the small number of patients the difference did not reach statistical significance $\left(\chi^{2}=1 \cdot 14\right.$, NS). A similar smaller difference occurred during week 2 . There was, however, a significant increase in recovery of klebsiella in the B7 CREG group at week 1 and week 2 when compared with control subjects $\left(\chi^{2}=12 \cdot 17, p<0.001\right.$, and $\chi^{2}=5 \cdot 3, p<0.025$ respectively) and when $B 7$ CREG patients from group A were compared with B7 CREG patients from group $C\left(\chi^{2}=5.71, p<0.025\right)$. There was no significant increase in klebsiella isolation from the B7 CREG negative patients during the first two

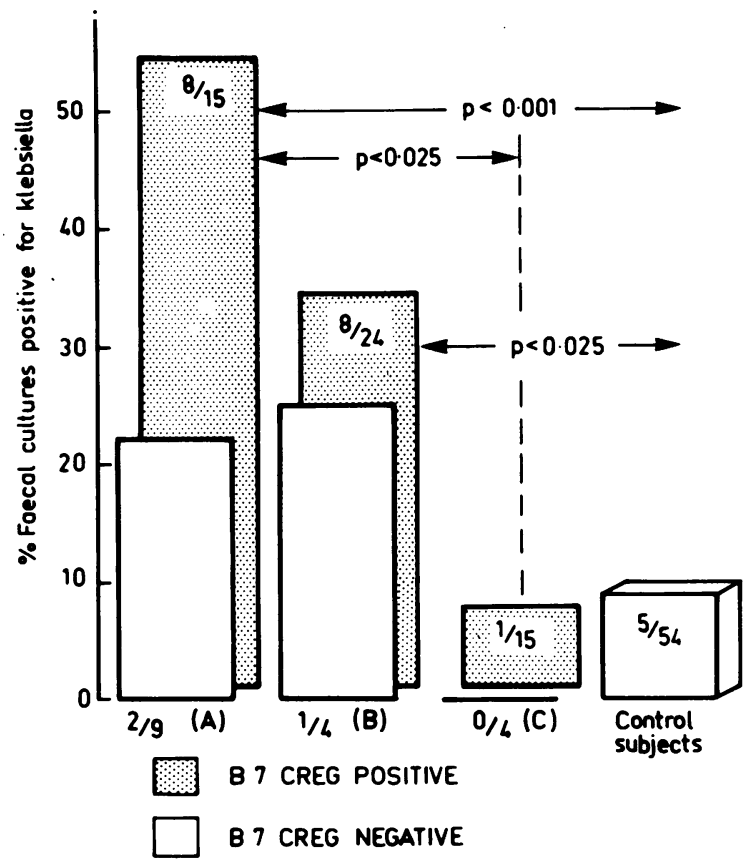

Fig. 2 Percentage faecal isolation rate of Klebsiella spp. in $B 7 C R E G(+)$ and $B 7 C R E G(-) A A U$ patients during the first $(A)$, second $(B)$, and third to fourth weeks $(C)$ after onset of symptoms and in controls.

weeks when compared with the control subjects, or with patients from weeks 3 and 4 .

ESR and klebsiella in AAU

The mean erythrocyte sedimentation rate (ESR) in AAU patients with faecal cultures positive for klebsiella was compared with that in patients having negative faecal cultures. Patients with positive faecal cultures had a higher mean ESR during both the first week $(26.1 \mathrm{~mm} / \mathrm{h}$ vs $12.5 \mathrm{~mm} / \mathrm{h}, t=1.66$, NS) and during the first and second week combined (24.5 $\mathrm{mm} / \mathrm{h}$ vs $15.3 \mathrm{~mm} / \mathrm{h}, t=2.04, \mathrm{p}<0.05$ ) (Fig. 3 ).

\section{Klebsiella in AAU patients with and without spondylarthritis}

Six of the 25 AAU patients seen in the first week had AS or a spondylarthritis-i.e., low back pain with sacroiliitis with or without peripheral arthritis. Four of these patients $(66 \%)$ had faecal cultures positive for Klebsiella spp. Although there was an overall increased isolation of Klebsiella spp. in spondylarthritic AAU patients $9 / 19$ (47\%) when compared with non-spondylarthritic patients $10 / 35$ $(29 \%)$ during the first two weeks, the differences between the two groups did not reach statistical significance $\left(\chi^{2}=1 \cdot 17, p>0 \cdot 05\right)$. The faecal isolation 


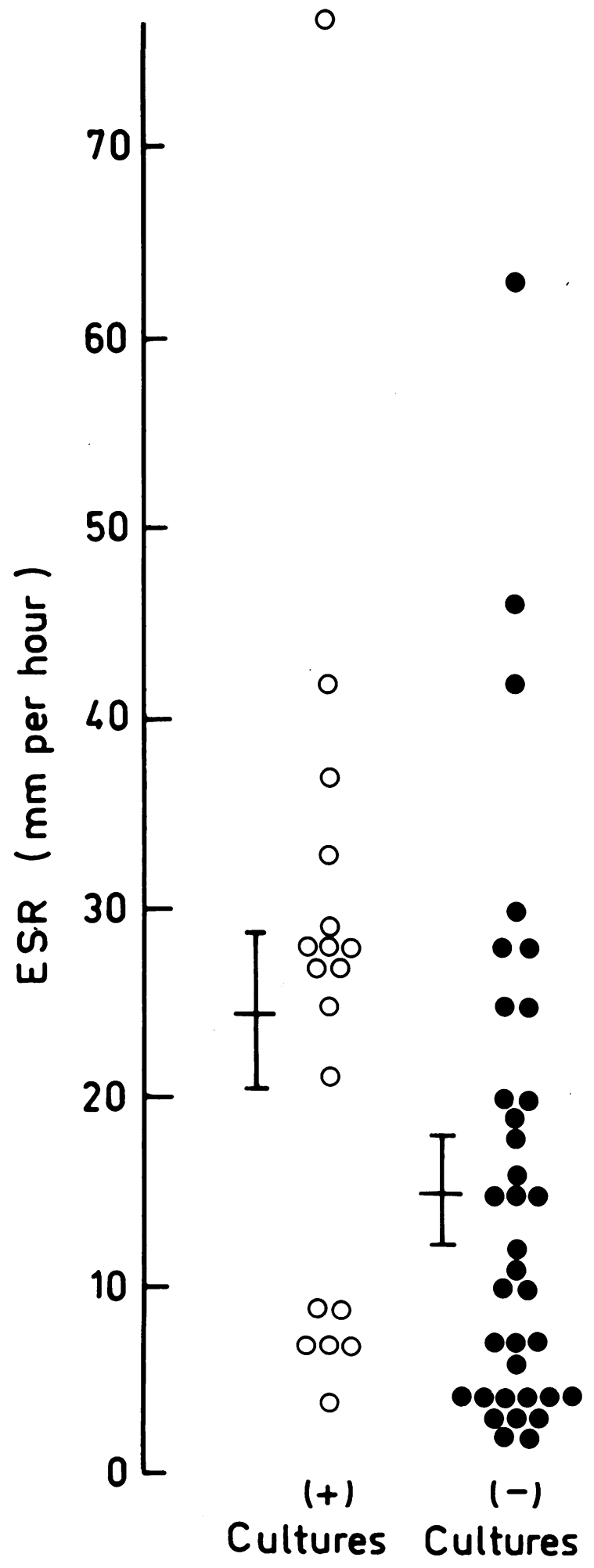

Fig. 3 Erythrocyte sedimentation rate in all $A A U$ patients with positive or negative faecal cultures for Klebsiella spp. seen within two weeks of onset of symptoms. Bars represent mean and SEM.

rate of klebsiella from AAU patients with spondylarthritis during the first two weeks combined was significantly greater than from the controls $\left(\chi^{2}=10.83, p<0.001\right)$, but there was also a significant increase in klebsiella recovery from nonspondylarthritic patients $\left(\chi^{2}=4.36, p<0.05\right)$ in comparison with the control group.

\section{Discussion}

Our results show that there is an increased recovery of Klebsiella species from the faeces of AAU patients in the first two weeks after onset of symptoms. After that time the faecal isolation rate is no different from the level found in a healthy control population. There is no evidence from our data to suggest that idiopathic AAU is associated with subclinical faecal carriage of salmonella, shigella, campylobacter, or yersinia.

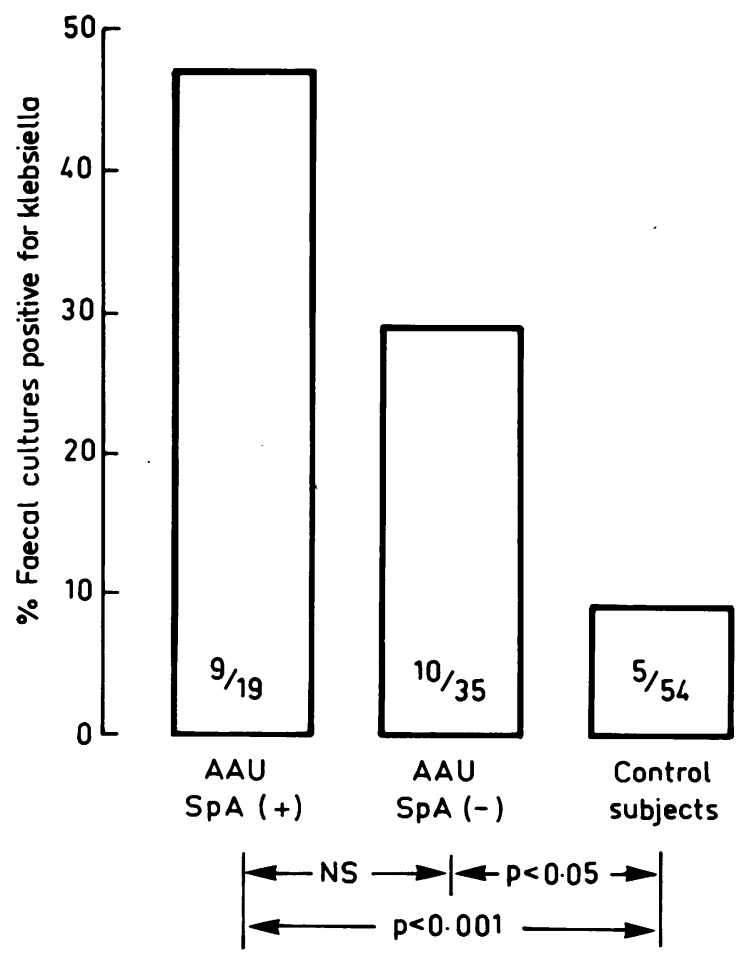

Fig. 4 Percentage faecal isolation rate of Klebsiella spp. in $A A U$ patients with spondylarthritis $S p A(+)$ and without spondylarthritis $S p A(-)$ seen within two weeks of onset of symptoms and in controls. 
The progressive decrease in faecal isolation rate of Klebsiella spp. in those patients seen at longer intervals after the onset of AAU is strongly suggestive of a temporal association between the presence of klebsiella in the gastrointestinal tract and the subsequent development of AAU. The $40 \%$ isolation rate in AAU patients seen within one week of onset of ocular symptoms can be interpreted in a number of ways. Firstly, klebsiella may be associated with only a proportion of cases. This is quite likely, as AAU is an ocular inflammatory condition which probably is initiated by numerous agents and has different pathogenetic mechanisms. Secondly, it is possible that the gastrointestinal carriage of klebsiella may even be higher than our figure of $40 \%$ in persons about to develop AAU. Only a prospective study of patients with recurrent attacks of AAU could resolve this question.

The decreasing faecal isolation rate of klebsiella in AAU patients seen at progressively later weeks after onset of symptoms suggests that there may be an immunological response to the presence of these micro-organisms. This hypothesis is supported by our recent observations of elevated levels of serum IgA in patients with AAU (paper in preparation). The elevated serum IgA was found predominantly in the HLA-B27 positive patient group.

Klebsiella recovery was higher from the HLAB27 $(50 \%)$ or HLA-B7 CREG $(53 \%)$ patients than from the B7 CREG negative patients $(22 \%)$ during the first week. The difference between the groups, however, was not significant. A similar trend was noted in the second week. It is possible that the numbers studied were too small. A larger continuing study now under way should answer this question. Willshaw examined 23 episodes of AAU and also noted an increased recovery of klebsiella in $6 / 10$ $(60 \%)$ B 27 positive patients as against $4 / 13(31 \%)$ in the B27 negative group. ${ }^{21}$ Again his small numbers failed to reach statistical significance. Our results are strikingly similar to his. Warren and Brewerton did not find an increased faecal recovery of klebsiella in AAU patients. ${ }^{19}$ Neither study, however, compared the time interval between the onset of patients' symptoms and the receipt of faecal cultures or compared B7 CREG positive AAU patients with B7 CREG negative patients.

The high klebsiella isolation rate from hospital staff was not unexpected. An increased faecal isolation rate of klebsiella from hospital staff is well recognised. ${ }^{21}$ The results obtained in our study are comparable with previously reported rates of isolation from hospital staff and indicate that our culture techniques are of comparable sensitivity. These results, however, highlight the relatively low klebsiella isolation rate obtained from healthy control subjects when they are selected from the general population, and the importance of selecting an appropriate control group. We obtained a similar result from a sample of physiotherapy students studied on their first day of contact with a hospital environment. ${ }^{17}$ In this respect it is important to note that the patients examined in our study were in effect 'non-hospital subjects,' having had only a single episode of minimal hospital exposure which would have been unlikely to affect the bowel flora.

The elevated mean ESR in patients with faecal cultures positive for klebsiella provides additional corroborative evidence that these organisms may have been producing significant systemic inflammatory disease. A similar association between klebsiella carriage, elevated ESR, and/or elevated serum C-reactive protein has been reported in AS. ${ }^{22.23}$

The mechanism by which klebsiella may produce AAU remains speculative. Circulating Gramnegative bacterial endotoxin can be shown to produce AAU in animal experiments. ${ }^{24}$ Intravitreal injection of Klebsiella oxytoca has been shown to produce a rapid destructive endophthalmitis with anterior segment inflammation. ${ }^{25}$ These observations would suggest a direct toxic effect by bacteria or bacterial cell products deposited in the eye.

Another mechanism is direct cross-reactivity between bacterial and ocular antigens. Crossreactivity between klebsiella antigens and bovine vitreous humour has been demonstrated, and it is possible that ocular inflammation may occur as a result of cellular and/or humoral mediated crossreactive (i.e. autoimmune) mechanisms. ${ }^{2627}$

The similarities between the increased klebsiella recovery rate in both HLA-B7 CREG associated AAU and acute exacerbations of inflammatory spine and peripheral joint disease in AS suggests that these two conditions may be different manifestations of a more general underlying disease process.

Infection with shigella, salmonella, and Yersinia enterocolitica can produce AAU, particularly in HLA-B27 positive persons. Our similar findings with klebsiella in HLA-B7 CREG positive AAU suggest that perhaps a number of Gram-negative enteric micro-organisms, possibly due to some common feature, such as a surface antigen, can produce AAU and/or arthritis in a genetically susceptible individual.

We are grateful to the many ophthalmologists, particularly Dr B. Coote, who referred patients to us for investigation. These studies were supported by a grant from the OPSM Foundation, the Austin Hospital Research Foundation, and a research project grant from the Royal Victorian Eye and Ear Hospital. We are also grateful to Janet Finlayson who typed the manuscript. 


\section{References}

1 James DG. Acute anterior uveitis. Clin Rheum Dis 1977; 3: 345-61.

2 Paronen I. Reiter's disease, a study of 344 cases observed in Finland. Acta Med Scand 1948; 131 (suppl 213): 1-114.

3 Saari KM, Vilpulla A, Lassus A, Leirisalo M, Saari R. Ocular inflammation in Reiter's disease after salmonella enteritis. $A m \mathrm{~J}$ Ophthalmol 1980; 90: 63-8.

4 Saari KM, Laitinen O, Leirisalo M, Saari R. Ocular inflammation associated with Yersinia infection. Am J Ophthalmol 1980; 89: 84-95.

5 Wakeficld D, Schrieber L, Penny R. Immunological factors in uvcitis. Med J Aust 1982; i: 229-31.

6 Brewerton DA. HL-A27 and acute anterior uveitis. Ann Rheum Dis 1975; 34 (suppl 1): 33-5.

7 Ebringer RW. Editorial review. HLA-B27 and the link with rheumatic diseases: recent developments. Clin Sci Mol Med 1980; 59: 405-10.

8 West SG, Tesar JT, Schwartz BD. Association of $X$ antigen with acute anterior uveitis (AAU). Arthritis Rheum 1982; 25 (suppl): abstr 356: 62.

9 Grizzard WS, Vasey FB, Perry LD, Espinoza LR. Cross reactive antigens of the $\mathrm{B} 7$ group (B7 CREG) in idiopathic uveitis. ARVO Abstracts 1982; 26: 214.

10) Ebringer A, Cowling P, Ngwa Suh N, James DCO, Ebringer RW. Cross reactivity between Klebsiella aerogenes species and B27 lymphocyte antigens as an actiological factor in ankylosing spondylitis (abstr). In: Dausset J, Svejgaard A, eds. HLA and disease. Paris: INSERM, 1976: 27.

11 Avakian H, Welsh J, Ebringer A, Entwistle CC. Ankylosing spondylitis, HLA-B27 and Klebsiclla II. Cross-reactivity studies with human tissue typing sera. Br J Exp Pathol 1980; 61: 865-7.

12 Geczy AF, Alexander K. Bashir HV, Edmonds J. A factor(s) in Klebsiella culture filtrates specifically modifies an HLA-B27associated cell-surface component. Nature 1980); 283: 782-4.

13 Ebringer RW, Cawdell D, Cowling P, Ebringer A. Sequential studies in ankylosing spondylitis: association of Klebsiella pneumoniae with active discasc. Ann Rheum Dis 1978; 37: 146-51.

14 Eastmond CJ, Calguneri M, Shincbaum R, Cooke EM, Wright
V. A sequential study of the relationship between faccal Klebsiella aerogenes and the common clinical manifestations of ankylosing spondylitis. Ann Rheum Dis 1982; 41: 15-20.

15 Kuberski TT, Morse HG, Rate RG, Bonnell MD. Increased recovery of Klebsiclla from the gastrointestinal tract of Reiter's syndrome and ankylosing spondylitis patients. Arthritis Rheum 1981; 24 (suppl 1, abstr 123): 78.

16 Warren RE. Brewerton DA. Faceal carriage of klebsiclla by patients with ankylosing spondylitis and rheumatoid arthritis. Ann Rheum Dis 1980; 39: 37-44.

17 Ebringer R. Cawdell D. Ebringer A. Klebsiella pneumoniae and acute anterior uveitis in ankylosing spondylitis. $\mathrm{Br}$ Med $\mathrm{J}$ 1979; i: 383 .

18 Willshaw HE, Eastmond CJ. Role of environmental factors in uveitis. Trans Ophthalmol Soc UK 1979; 99: 160-2.

19 Warren RE, Brewerton D. Klebsiella, spondylitis, and uveitis. Br Med J 1979; i: 889 .

20) Willshaw HE. Acute anterior uveitis and Klebsiella aerogenes: a casual relationship. Br J Ophthalmol 1981: 65: 796-7.

21 Selden R. Lee S. Wang WLL, Bennet JV, Eickhofft C. Nosocomial Klebsiclla infections: intestinal colonization as a reservoir. Ann Intern Med 1971; 74: 657-64.

22 Cowling P, Ebringer R, Cawdell D, Ishii M, Ebringer A. Creactive protein, ESR, and klebsiclla in ankylosing spondylitis. Ann Rheum Dis 1980; 39: 45-9.

23 Hunter T, Harding GKM, Kaprove RE, Schroeder ML. Faceal carriage of various Klebsiclla and Enterobacter species in patients with active ankylosing spondylitis. Arthritis Rheum 1981: 24: 106-8.

24 Rosenbaum JT, McDevitt HO, Guss RB, Eghert PR. Endotoxin-induced uveitis in rats as a model for human discasc. Nature 1980; 286: 611-3.

25 Meyers-Elliot RH, Dethlefs BA. Experimental Klebsicllainduced endophalmitis in the rabbit. Arch Ophthalmol 1982; 100: $1959-63$.

26 Avakian H, Abuknesha R, Welsh J, Ebringer A. Uveitis, vitreous humour, and klebsiclla. I. Binding studies with rabbit antisera. Br J Ophthalmol 1981; 65: 315-22.

27 Welsh J. Avakian H, Ebringer A. Uveitis, vitreous humour and klebsiclla. II. Cross-reactivity studics with radioimmunoassay. BrJ Ophthalmol 1981; 65: 323-8. 